\title{
Automated Microscopy: Macro Language Controlling a Confocal Microscope and its External Illumination: Adaptation for Photosynthetic Organisms
}

6 Gábor Steinbach ${ }^{\star}$ and Radek Kaňa

\author{
Institute of Microbiology, Academy of Sciences, Centrum Algatech, Novohradska 237 - Opatovicky mlýn, CZ 37901 Třeboñ, \\ Czech Republic
}

Key words: automated microscopy, remote controlled microscopy, confocal microscopy, photosynthetic membrane, photoprotection

\section{INTRODUCTION}

Upgrading confocal microscopes can initiate new directions in subcellular biology research, moreover, biologists participating in the development can express their needs to establish new functions for microscopes (White et al., 1987; Amos, 2000). Some of the required methods are labor-intensive-especially the acquisition of long-term time series. Difficulties arise when the imaging work requires nonperiodic sampling, e.g., collecting images on a logarithmic timescale, or with variable measurement parameters. Most vendors can provide partial solutions for building a specific protocol (e.g., Olympus: Time Controller or Zeiss: Experiment Designer) and there are also dedicated solutions for automated microscopy (e.g., imaging machines of Aquifer). These solutions can spare human resources and make high-content screening measurements possible. In most systems, construction of the custom measurement protocol is based on a graphical environment. This is convenient for less-experienced users; however, there are disadvantages when using graphical user interfaces (GUIs). Changing protocols quickly and flexibly or generating

${ }^{*}$ Corresponding author. steinbach@alga.cz protocols by external programs is highly limited. Further problems emerge when additional functions are required, especially communication with external hardware, e.g., switching between samples, or providing physical or chemical treatments. Third-party solutions have been developed in order to bypass these problems, e.g., for Zeiss microscopes (Yokoo et al., 2015).

Specific light conditions are often required to study the physiology of phototrophic microorganisms (Yokono et al., 2015). These organisms (cyanobacteria, algae, and higher plants) employ light-dependent photosynthesis as the main energy source for their metabolism. In fact, the efficiency of photosynthesis and photosynthetic rate are highly dependent on irradiance. This can be seen in the light-dependency curves of various photosynthetic parameters, including the photochemical efficiency of photosystems or $\mathrm{CO}_{2}$ assimilation rates (Papageorgiou \& Govindjee, 2004). Moreover, not only light quantity, but also light "quality" (wavelength) is an important factor shown in the action spectra of photosynthesis or the Emerson effect-an early evidence for the existence of two photosystems (Emerson, 1957).

Protocols examining specific changes in light quality (wavelength) and quantity are the main experimental approaches to study the mechanism of photosynthesis in cell suspension. Light- (and heat-) induced changes have been 
described for photosynthetic antenna systems (Stoitchkova et al., 2007; Szabo et al., 2008; Kaňa et al., 2009) as excessive light or heat are stress factors affecting photosynthesis on several levels (Kaňa et al., 2008; Cheregi et al., 2015). To resolve these mechanisms in more detail, we need to study light/heat effects on photosynthesis at the single-cell level in vivo by confocal measurements. The epifluorescence microscopy set-up for whole cell measurements has already been developed (Kupper et al., 2000), however, the system for confocal imaging has not been developed. Our newly developed macro language-controlled system for a confocal microscope overcomes most of the limitations of the original microscope controller GUIs and allows users to study the physiology of photosynthesis [e.g., mechanism of photoprotection in cyanobacteria (Kirilovsky et al., 2014)] under variable light conditions.

\section{Materials And Methods}

\section{Software Module}

The Cell $\oplus$ Finder extension system has been developed for Olympus FV1000 and FV1200 confocal microscopes and tested with FluoView 4.0b and 4.1a software versions. The code was written in C, and compiled with Pelles C 8.00.60 (Orinius, 2015). The minimum system requirement for Cell $\oplus$ Finder is Microsoft Windows XP but it works under Windows 7,8 , and 10 as well.

Communication between the Cell $\oplus$ Finder and the FluoView programs used the Microsoft Windows standard messaging system for window procedures (http://dev. windows.com/en-us/desktop). It is independent of the position of the windows: communication does not rely on the actual coordinates of the programs on the screen and the keyboard/mouse are not in any way blocked or disabled. (The original functions of the microscope were fully available while using Cell $\oplus$ Finder, even while a macro was running.)

Here, the initialization routine scans the connections to FluoView software elements and provides information for the higher level routines (like macro commands and mouse actions). An optional initialization process establishes connection to the microcontroller (Fig. 1).

\section{Hardware Module}

The attached microcontroller was an ATMega328 chip (Atmel Corp., San Jose, California, CA, USA) on an Arduino Nano board (Arduino LLC, Somerwille, MA, USA) (https:// www.arduino.cc), and communication was performed through a Universal Serial Bus, using a Future Technology Devices International Limited (Glasgow, United Kingdom) virtual COM port. The connection was established in a fully automated way (the port number was determined by the Cell $\oplus$ Finder program without user interaction or ini file settings). Communication between the Cell $\oplus$ Finder program and the microcontroller was based on short ASCII commands with optional integer parameters. The Arduino

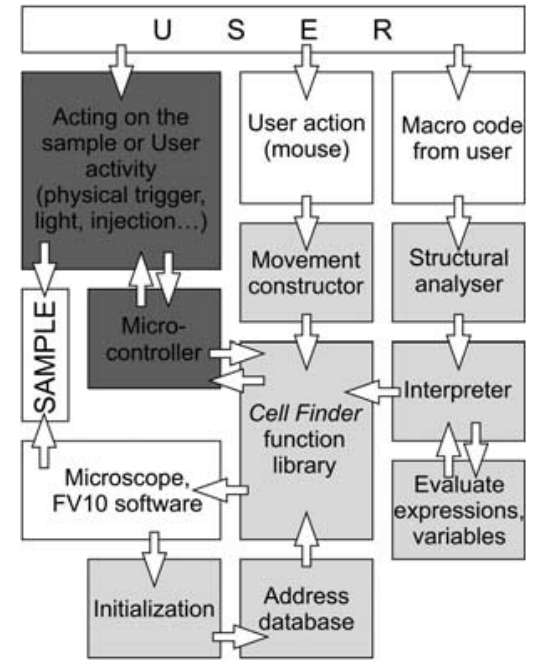

Figure 1. The connection scheme of the software modules and hardware elements of the microscope and Cell $\oplus$ Finder: The user provides mouse actions and macro code: they determine the communication and the commands to send by Cell $\oplus$ Finder to the microscope software and to the microcontroller. By initialization a database is built for the current addresses of the controls in FV10 - the standard library functions are based on this information. Elements of Cell $\oplus$ Finder are gray (software: light, hardware: dark). Arrows represent connection and data flow between the units.

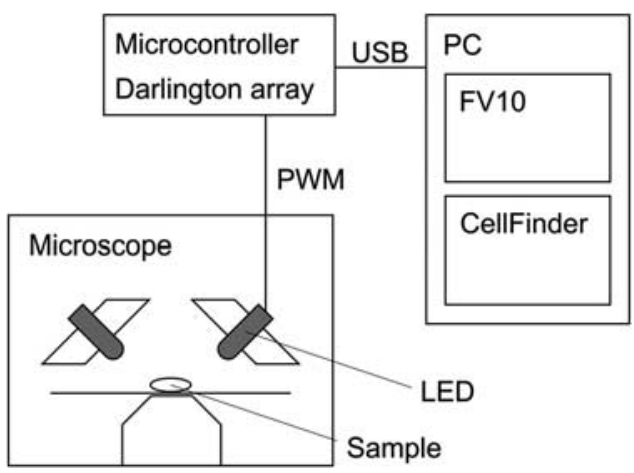

Figure 2. Scheme of the measurement: Cell $\oplus$ Finder's microcontroller part attached to the computer via USB. It controls the LED light source by $5 \mathrm{~V}$ PWM signal. The LEDs are held by an aluminum cone in a black box providing dark adaption between the image acquisitions. USB, Universal Serial Bus; PWM, pulsewidth modulation; LED, light emitting diode.

board itself did not initiate communication, but for handshaking it responded to certain signals from the computer. Most of the commands from the computer were executed without sending a reply by the Arduino on the serial communication line.

The Arduino Nano board provided 12 general input/ output digital pins of which six could be used for pulse-width modulation (PWM) signals in order to set certain light intensities or to drive servo motors. Eight analogue input channels were also available, e.g., for thermal sensors. The current version of Cell $\oplus$ Finder supports three PWM 
outputs, three digital trigger inputs, and three analogue input channels, but it is easy to extend the communication language for more input/output channels, even for other Arduino boards (e.g., the "Mega" board provides 12 PWM output pins).

External illumination was provided by eight orange light emitting diodes (LEDs) (peak wavelength: $640 \mathrm{~nm}$ ), which were held in place by a conical aluminum block in order to focus light to one spot in the center of the field of view (Fig. 2). Light intensity was adjustable from 0 to $380 \mu \mathrm{mol}$ photons $/ \mathrm{m}^{2} / \mathrm{s}$ using the PWM output of the microcontroller and a Darlington transistor array (ULN2003APG, Toshiba America Inc., New York, NY, USA). Digital trigger inputs can be used in the Cell $\oplus$ Finder macro (as built-in variables) or the other pins can be directly used by the microcontroller (read/write).

\section{Cell Cultures}

Synechocystis sp. PCC6803 cells were cultivated in BG 11 medium $\left(\right.$ at $\left.28^{\circ} \mathrm{C}\right)$ under continuous white light (fluorescent tubes, $40 \mu \mathrm{mol}$ photons $/ \mathrm{m}^{2} / \mathrm{s}$ ). For imaging, the living cells were centrifuged three times for $5 \mathrm{~min}$ at $8,000 \mathrm{rpm}$, and the pellet was resuspended in the growth medium.

\section{Confocal Fluorescence Microscopy}

Cell $\oplus$ Finder was developed and applied for an Olympus FV1000 confocal laser scanning microscope. A UAPON 100X OTIRF NA: 1.49 objective was used during the measurements. Chlorophyll fluorescence was excited by the $488 \mathrm{~nm}$ Ar-ion laser line (power: 10\%), fluorescence emission was detected between 690 and $790 \mathrm{~nm}$. The phycobilisome (PBS) fluorescence was induced by a $635 \mathrm{~nm}$ diode laser (power: 0.1\%), PBS fluorescence was detected between 650 and $680 \mathrm{~nm}$. Pinhole size was $175 \mu \mathrm{m}$. Images contained $800 \times 800$ pixels, taken at a speed of $4 \mu$ s/pixel.

\section{Test Protocol}

To demonstrate the use of Cell $\oplus$ Finder, the following "illuminate and measure" protocol was applied. The cells were dark adapted for 10 min before measurement. The sequence of the macro was as follows:

1. Define the calibration variable for the light intensity determination.

2. Prepare the microscope for chlorophyll fluorescence imaging. Take an image.

3. Prepare the microscope for PBS fluorescence imaging. Take an image.

4. Repeat acquisitions 2 and 3 once (in order to double-check that the sample did not drift between switching channels).

5. Set the light to $380 \mu \mathrm{mol}$ photons $/ \mathrm{m}^{2} / \mathrm{s}$ intensity.

6. Wait $4 \mathrm{~min}$.

7. Generate an alarm sound to allow the user to reset the position of the sample, if required. The mouse functions of Cell $\oplus$ Finder provide an easy way to correct any unwanted motion.
8. Wait $1 \mathrm{~min}$ (so the user has time to move the sample, if needed).

9. Generate an alarm sound $10 \mathrm{~s}$ before the next imaging sequence (so the user is warned that an imaging sequence is about to commence).

10. Switch off the orange light.

11. Restart the macro from the second line: perform imaging again.

This macro provided images for $90 \mathrm{~min}$, the only user input needed was to verify the position of the cells when the alarm signal sounded. (The macro code is available on the download page of the program: http://www.alga.cz/cellfinder)

\section{Results}

\section{Enhanced User Environment}

Our software facilitates an easy and intuitive way to locate the appropriate area for imaging using the mouse. By clicking the left mouse button, it was possible to pan (move) the field of view. The moving function is always matched with the visible area in the "Live View" window of the Olympus software-regardless of the zoom factor. The movement was rescaled by the actual zoom setting.

The zoom factor could be adjusted using the mouse wheel. This was not continuous, it worked based on predefined steps (e.g., on a quasi-logarithmic scale-defined in the Settings of the program). This enabled a quick overview of the sample on a wide range of zooms. Moreover, changing the zoom factor in discrete steps ensured that the acquired images were comparable (with a uniform scale) even when very many images were collected.

As a second function, the mouse wheel could be used to move the objective in the $Z$ direction by small (predefined) steps within predefined limits (defined in Settings of the program). Moving the mouse wheel with the right button released controls the zoom factor, whereas moving the wheel with the right button depressed moves the objective along the $Z$ axis.

In focusing (preview) mode, the Cell $\oplus$ Finder software automatically switched the laser and the scanning function on/off according to the mouse actions and predicts when the laser is needed. This way it minimalized both the bleaching of the sample and the number of times the focusing mode was switched on/off.

These enhanced mouse functions provided a more convenient working environment. Thus, the premeasurement period was reduced and sensitive fluorescent samples were protected from bleaching. Using these special mouse functions helped to keep the cells in position for the $90 \mathrm{~min}$ of the test measurement. Small shifts in the sample were easily corrected, using the mouse with a movement of the same length as the shift of the cells visible on the screen.

\section{Macro Language}

The macro interpreter part of the software controls the measurement routine. Timing, image acquisition and 
Table 1. Commands for the Macro Language.

\begin{tabular}{|c|c|}
\hline Command Parameters & Descriptions \\
\hline A int & $\begin{array}{l}\text { Acquisition, then wait int ms (e.g., for bleaching) - before that it waits for the acquisition time }+ \text { some safety } \\
\text { period. Thus, the total time for the statement: }<\text { AcqTime }>+i n t \mathrm{~ms}+2.5 \mathrm{~s}+\text { speed (see timing commands) }\end{array}$ \\
\hline M laserpower int float & The number of laser or wavelength $(405 / 458 / 488 / 515 / 559 / 635)$, power in percentages \\
\hline M lasersoff & Set all lasers to $0 \%$ \\
\hline M blaserpower int float & $\begin{array}{l}\text { The number of bleaching laser or wavelength }(405 / 458 / 488 / 515 / 559 / 635) \text {, power in percentages-for the main } \\
\text { scanner bleaching }\end{array}$ \\
\hline M blasersoff & Set all main scanner bleaching lasers to $0 \%$ \\
\hline M hvchs1 I hvchs2 int & High voltage for detectors in fluorescence channels 1 and 2 (chs1/chs2) \\
\hline M wlchs1 I wlchs2 int int & Spectral selection for chs1/chs2: start and width \\
\hline $\mathrm{P}$ relx int & Move pan in microscope's "um” units (relative) \\
\hline \multicolumn{2}{|r|}{ 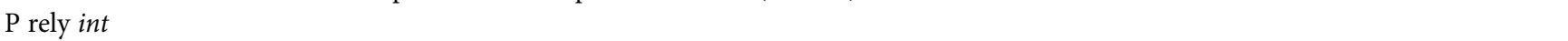 } \\
\hline $\mathrm{P}$ setunit int & Set the screen size in microscope's "um" units, default: 100 \\
\hline $\mathrm{P}$ autosetunit $[$ int $]$ & $\begin{array}{l}\text { Set the screen size according to the } \text { zoom }^{\text {a }} \text { (with some overlapping). The int can rescale it (in \%) to have more or } \\
\text { less overlapping }\end{array}$ \\
\hline P uabsx I uabsx int & Move pan in preset screen units (absolute) \\
\hline P urelx I urely int & Move pan in preset screen units (relative) \\
\hline P tile int & Move to the numbered tile position (box size from preset unit) max 80 \\
\hline P starttile & Move to the first tile \\
\hline P nexttile & Move to the next tile \\
\hline P zoom float & Set zoom factor \\
\hline P zoomin I zoomout & Using the predefined zooms \\
\hline B long I short I end & Predefined beeps \\
\hline B question I error I ding & Windows sounds \\
\hline B int int & $\mathrm{Hz}$ ms - user defined beep \\
\hline $\mathrm{D}$ end I quit text & Stop running, print text \\
\hline D gotonum int & Continue from the specified line \\
\hline D gotolabel name & Continue from the specified label \\
\hline D label name & Set a label \\
\hline D setmaxjump int & Maximal number of goto jumps, default: 20 \\
\hline $\mathrm{D}$ resetjump & Restart counting \\
\hline D gosub name & Continue from the specified label \\
\hline D return & Return to the gosub call \\
\hline L text & Write text to $\log$ \\
\hline $\mathrm{L}<$ newline $>$ & Print a new line \\
\hline $\mathrm{L}<$ calc $>$ expression & Evaluate the expression and print to $\log$ \\
\hline $\mathrm{L}<$ calc_> expression & Evaluate the expression without printing (assignments usually) \\
\hline $\mathrm{L}<$ clear $>$ & Clear log \\
\hline \# I; remark & Not executed \\
\hline S int & Speed in ms, default: $1,000 \mathrm{~ms}$ \\
\hline S teston I testoff & Slow down execution/normal run \\
\hline S starttimer & Start counting (in ms) for RunTime variable \\
\hline $\mathrm{W}$ int & Waiting time in $\mathrm{ms}$ \\
\hline
\end{tabular}

${ }^{\text {aIf }}$ you change the zoom, you have to execute again.

acquisition parameters were set by the macro interpreter It announced possible user interaction requests, calculated light intensity (a fully equipped mathematical calculator routine was also part of the syntax of the language, see the documentation: http://www.alga.cz/cellfinder) and controlled switching of the illumination on and off between imaging periods. The available commands can be divided into four groups: (1) acquisition and setting microscope parameters, (2) positioning the sample, (3) user communication (log and sounds), and (4) directives (jumps, subroutines, cycles) (Table 1).

\section{Hardware Extension}

The manufactured hardware extension provided illumination synchronized with the imaging: switching on between each fluorescent scan. The calibration value for the proper light intensities was stored as a variable in the macro-based on the output value of the standard Photosynthetically Active Radiation meter (Li-Cor LI-250A; LI-COR Inc., Lincoln, NE, USA) at full strength of the LEDs. Illumination is fully synchronized and provided between acquisition of images. 


\section{Results of Applied Protocol: PBS Decoupling}

PBS fluorescence intensity has been detected during long-term irradiation with orange light $(635 \mathrm{~nm}$, intensity $380 \mu \mathrm{mol}$ photons $\mathrm{m}^{-2} / \mathrm{s}$ ), which is absorbed by PBSs. Total PBS fluorescence inside a single cell almost doubled on average during the whole illumination period $(90 \mathrm{~min}$; see Fig. 3). The fluorescence increase started at $\sim 1 \mathrm{~h}$ after excessive illumination treatment. It has been proposed that PBS decoupling from photosystems can cause an increase in PBS fluorescence, as excitation energy is not transferred to the photosystems (Kaňa et al., 2009; Tamary et al., 2012; Chukhutsina et al., 2015). Hence, PBS decoupling could be a photoprotective mechanism for cyanobacteria (see review by Kirilovsky et al., 2014). The kinetic data obtained with the Cell $\oplus$ Finder tool shows that the process of PBS decoupling appears after long-term exposure to light. Thus, it is demonstrated that this process may have biological relevance only in the case of long-term light stress.

\section{DiscusSiON}

The Cell $\oplus$ Finder program solved several technical difficulties in performing special time-lapse measurements: communicating with the user and with external devices using flexible methods, as well as dealing with sensitive samples. The system can be modified/extended in order to suit various needs. Any future third-party development that makes use of the program in more areas is welcome. Future developments may include moving the sample using the motorized stage of the microscope or some additional macro functions (e.g., more flexible cycles). In fact, projects like this are never completely finished: the evolution of the program will answer the challenges of new applications.

This system has been developed for Olympus confocal microscopes (FV1000 and FV1200; Olympus Corp., Tokyo, Japan), however, it is open to be adapted to any other microscope systems by changes in the program code or by way of using the concept of Cell $\oplus$ Finder for developing a new software/hardware system specialized for other confocal microscope systems. This study presents a simple and practical tool for performing special microscopy applications with an Olympus system and is also a proof-of-concept in the field of specialized microscopy. It initiates technical/ software progress allowing connection between external instruments and confocal microscopes using improved device-device communication.

Cell $\oplus$ Finder as a hardware/software solution opens a new field in photosynthesis research when photosynthetic activity can be study on the subcellular level. In fact, our approach allowed us to study detailed mechanisms of photoprotection in single-celled cyanobacterium (see e.g., review Kirilovsky et al., 2014). The image data indicates that the process of PBS decoupling (shown by the increase in PBS fluorescence, see Fig. 3) is stimulated only slowly, as the response to illumination was seen only after a minimum of
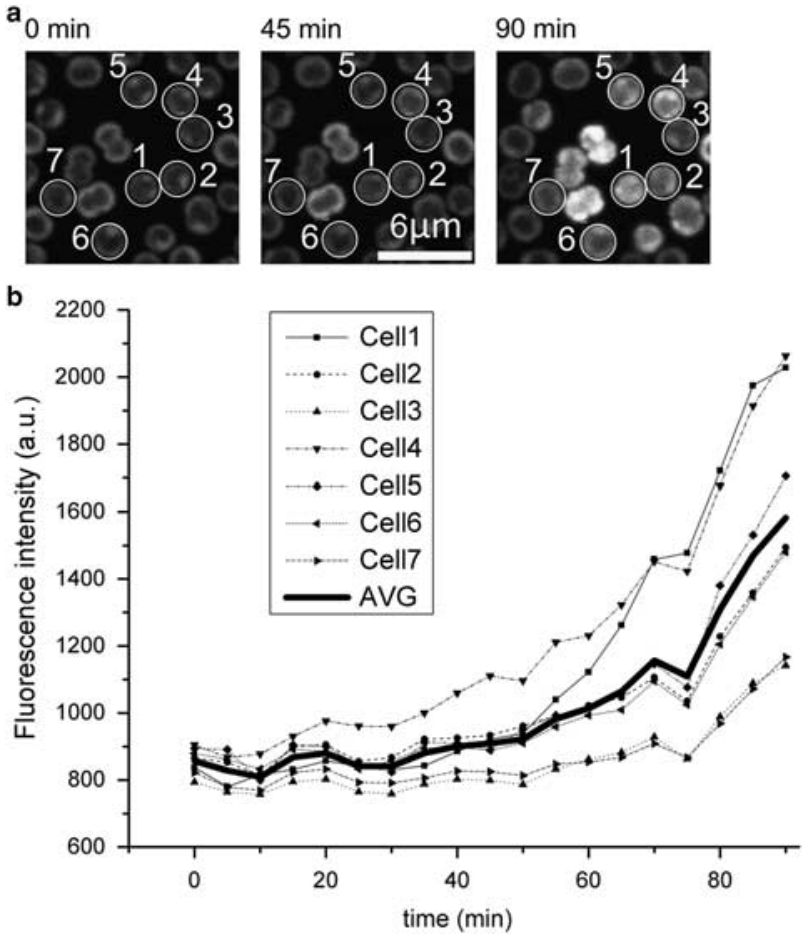

Figure 3. The red illumination of the PBSs initiates a decoupling from the photosystems: the fluorescence intensity increases over time. Imaging every 5 minutes, excitation $635 \mathrm{~nm}$, emission: $650-680 \mathrm{~nm}$.

$1 \mathrm{~h}$ of irradiation. This contrasts with recent results proposing PBS uncoupling from photosystem I during the dark-light transition (Chukhutsina et al., 2015). This discrepancy may be explained either by different experimental conditions (compare white light used by Chukhutsina et al., 2015 with our orange light), and/or by our single-cell approach. Indeed, heterogeneous behavior in different cell microdomains has been observed (compare membrane area/non-membrane area in Fig. 3). We have recently described these specialized bioenergetics microdomains in cyanobacteria (Steinbach et al., 2015). Therefore, the single-cell measurements together with specialized irradiation (both provided by our system) are necessary to explore the mechanisms of PBS decoupling in the future. Our system is able to open a new area of photosynthesis research, where heterogeneity in photosynthetic functions will be studied on a single-cell level.

\section{CONCLUSION}

With the Cell $\oplus$ Finder, sensitive samples can be imaged in a more user- and cell-friendly way. This third-party tool for confocal microscopy in photosynthesis research or in other applications increases the range of possible (semi-)automated measurements, and this system provides an efficient tool for high throughput screening research. The hardware components can be modified easily (since they are well documented) for any future needs. 


\section{ACKNOWLEDGMENTS}

This work was supported by projects of the Czech Science Foundation (P501-12-0304) and by the Ministry of Educationproject Algain (EE2.3.30.0059) and by institutional projects Algatech (CZ.1.05/2.1.00/03.0110) and Algatech Plus (MSMT LO1416). The authors would like to thank Judith Noda for cell cultivation and Jiři Šetlik for constructing the light source.

\section{REFERENCES}

Amos, B. (2000). Lessons from the history of light microscopy. Nat Cell Biol 2(8), E151-E152.

Cheregi, O., Kotabová, E., Prášll, O., Schroder, W.P., Kaña, R. \& Funk, C. (2015). Presence of state transitions in the cryptophyte alga Guillardia theta. J Exp Bot 66(20), 6461-6470.

Chukhutsina, V., Bersanini, L., Aro, E.-M. \& Van Amerongen, H. (2015). Cyanobacterial light-harvesting phycobilisomes uncouple from photosystem i during dark-to-light transitions. Sci Rep 5(14193), 1-10.

EMERSON, R. (1957). Dependence of yield of photosynthesis in longwave red on wavelength and intensity of supplementary light. Science 125(3251), 746.

KaŇa, R., Kotabova, E. \& Prášll, O. (2008). Acceleration of plastoquinone pool reduction by alternative pathways precedes a decrease in photosynthetic $\mathrm{CO}(2)$ assimilation in preheated barley leaves. Physiol Plant 133(4), 794-806.

Kaña, R., Prášst, O., Komárek, O., Papageorgiou, G.C. \& Govindjee (2009). Spectral characteristic of fluorescence induction in a model cyanobacterium, Synechococcus sp (PCC 7942). Biochim Biophys Acta 1787(10), 1170-1178.

KIRILOVSKY, D., KaŇA, R. \& PrÁšLL, O. (2014). Mechanisms modulating energy arriving at reaction centers in cyanobacteria. In NonPhotochemical Quenching and Energy Dissipation in Plants, Algae and Cyanobacteria, Demmig-Adams, B., Garab, G., Adams III, W. \& Govindjee (Eds.), pp. 471-501. The Netherlands: Springer.

Kupper, H., Šetlík, I., Trtílek, M. \& Nedbal, L. (2000). A microscope for two-dimensional measurements of in vivo chlorophyll fluorescence kinetics using pulsed measuring radiation, continuous actinic radiation, and saturating flashes. Photosynthetica 38(4), 553-570.

Orinius, P. (2015). Pelles C. http://pellesc.com/

PAPAgEORgIOU, G.C. \& GovindjeE (2004). Chlorophyll a Fluorescence: A Signature of Photosynthesis. The Netherlands: Springer.

Steinbach, G., Schubert, F. \& KaŇA, R. (2015). Cryo-imaging of photosystems and phycobilisomes in Anabaena sp. PCC 7120 cells. J Photochem Photobiol B 152, 395-399.

Stoitchkova, K., Zsiros, O., Javorfi, T., Pali, T., Andreeva, A., Gombos, Z. \& Garab, G. (2007). Heat- and light-induced reorganizations in the phycobilisome antenna of Synechocystis sp PCC 6803. Thermo-optic effect. Biochim Biophys Acta 1767(6), 750-756.

Szabo, M., Lepetit, B., Goss, R., Wilhelm, C., Mustardy, L. \& GARAB, G. (2008). Structurally flexible macro-organization of the pigment-protein complexes of the diatom Phaeodactylum tricornutum. Photosynth Res 95(2-3), 237-245.

Tamary, E., Kiss, V., Nevo, R., Adam, Z., Bernat, G., Rexroth, S., Rogner, M. \& Reich, Z. (2012). Structural and functional alterations of cyanobacterial phycobilisomes induced by high-light stress. Biochim Biophys Acta 1817(2), 319-327.

White, J.G., Amos, W.B. \& Fordham, M. (1987). An evaluation of confocal versus conventional imaging of biological structures by fluorescence light-microscopy. J Cell Biol 105(1), 41-48.

Yokono, M., Takabayashi, A., Акimoto, S. \& Tanaka, A. (2015). A megacomplex composed of both photosystem reaction centres in higher plants. Nat Commun 6, article no. 6675.

Yokoo, R., Hood, R.D. \& Savage, D.F. (2015). Live-cell imaging of cyanobacteria. Photosynth Res 126(1), 33-46. 